\title{
Ultrasound-induced Microbubble Coalescence
}

\author{
Michiel Postema,${ }^{* \dagger}$ Philippe Marmottant,${ }^{\ddagger}$ Charles T. Lancée, ${ }^{*}$ \\ Sascha Hilgenfeldt, ${ }^{\ddagger}$ NiCO DE JONG ${ }^{* \dagger \uparrow}$ \\ *Department of Experimental Echocardiography, Thoraxcentre, Erasmus MC, Rotterdam, The \\ Netherlands; ${ }^{\dagger}$ Interuniversity Cardiology Institute of the Netherlands, Utrecht, The Netherlands; \\ and ${ }^{\ddagger}$ Physics of Fluids, Department of Applied Physics, Faculty of Science and Technology, \\ University of Twente, Enschede, The Netherlands
}

\S. Hilgenfeldt and N. de Jong share last author responsibility.

\begin{abstract}
We study the interaction of ultrasound contrast agent bubbles coated with a layer of lipids, driven by $0.5 \mathrm{MHz}$ ultrasound. High-speed photography on the sub-microsecond timescale reveals that some bubbles bounce off each other, while other show very fast coalescence during bubble expansion. This fast coalescence cannot be explained by dissipation-limited film drainage rates. We conclude that the lipid shell ruptures upon expansion, exposing clean free bubble interfaces that support plug flow profiles in the film and inertia-limited drainage whose timescales match those of the observed coalescence.
\end{abstract}

Short title: Microbubble Coalescence 
Key words: Microbubble coalescence, ultrasound contrast agent, film drainage, high-speed photography

\section{Introduction}

Ultrasound contrast agent (UCA) is a suspension of encapsulated gas bubbles. The bubbles have diameters ranging from 1 to $10 \mu \mathrm{m}$. They oscillate upon insonification, generating a characteristic acoustic response. Contrast agents are widely used in medical diagnostics (Burns et al., 1990; Goldberg et al., 2001).

Observing ultrasound insonified UCA with a high-speed camera is a promising method for analyzing microbubble oscillation and destruction behavior (Takeuchi, 1999; de Jong et al., 2000; Chomas et al., 2001; Kudo et al., 2002; Postema et al., 2003). Expanding UCA microbubble coalescence, observed with a high-speed camera during one cycle of ultrasound, has been previously reported by us (Postema et al., 2003). Colliding bubble coalescence has been studied with a camera by Kok (1993) and van Wijngaarden (1993). In the same year, Kumaran \& Koch (1993) computed how bubbles may repeatedly collide with each other.

To understand microbubble coalescence, one needs to comprehend the drainage of the liquid separating the bubble surfaces. Reynolds (1886) noted that the viscosity of a liquid can be determined by pressing two flat plates 
together, squeezing the liquid out, and measuring the drainage velocity. Thus, he formulated an equation for the drainage velocity of a fluid between rigid surfaces. General theories on the coalescence of colliding bubbles and droplets that are based on liquid film drainage, were put forward by Marrucci (1969), Dimitrov \& Ivanov (1978), Ivanov et al. (1979), Lin \& Slattery (1982a), Chesters \& Hofman (1982), Duineveld (1994a), and Klaseboer et al. (2000). Literature overviews on film drainage and bubble coalescence were given by Kralchevsky et al. (1996), Narsimhan \& Ruckenstein (1996), and Dhainaut (2002).

Theories on droplet coalescence find applications in fuel ignition research and aerosol studies, whereas the research on bubble coalescence focuses on thin film physics and foam stability (Kralchevsky et al., 1996; Narsimhan \& Ruckenstein, 1996). This paper explores ultrasound-induced coalescence of microbubbles. Controlled coalescence has potential applications in the clinical field.

Theories on bubble coalescence are generally based on the collision of unencapsulated bubbles or droplets, approaching each other at constant velocity. During expansion, microbubbles may also come into contact with each other, resulting in coalescence or bounce. We discriminate the following stages in the coalescence mechanism, optically observed in Fig. 1 and schematically represented in Fig. 2. These stages are similar to those presented for colliding bubbles and droplets in (Dhainaut, 2002). First, two bubbles approach collision while expanding (Fig. 2a). Prior to contact, there may be a flattening of the 
adjacent bubble surfaces, trapping liquid in between (Fig. 1a, Fig. 2b). This trapped liquid drains (Fig. 1b, Fig. 2c) until the separation reaches a critical thickness (Fig. 2d). An instability mechanism (Fig. 2d, magnified) results in rupture of the separation (Fig. 2e) and the formation of a merged bubble (Fig. 1c). After coalescence the resulting bubble will have an ellipsoidal shape (Fig. 1d, Fig. 2f). Owing to surface tension, it will relax to a spherical shape. When the contact time is less than the time needed for film drainage, the bubbles bounce off each other (Chaudhari \& Hofmann, 1994). We define bubble coalescence as the fusing of two or more bubbles into a single bubble. The process begins with the flattening of the bubble surfaces and is considered finished when the resulting bubble has a spherical shape.

In this paper, we give a description of the coalescence of expanding microbubbles, based on optical observations and theoretical modeling. First, we present theories for expanding bubble coalescence, based on film drainage theory. Then, we present experimental results, obtained by recording optical images of insonified UCA. Finally, theories and results are compared and discussed. 


\section{Theory}

\section{Flattening of the interface}

Flattening of the opposing bubble surfaces occurs because the liquid inertia overcomes the capillary pressure, as described in earlier work on colliding bubbles with constant volumes. For colliding bubbles, flattening happens if the bubble system has a Weber number We $\approx 0.5$ (Chesters \& Hofman, 1982; Duineveld, 1994b). The Weber number for two colliding bubbles with radii $R_{1}$ and $R_{2}$, respectively, is given by the inertial force relative to the surface tension force:

$$
\mathrm{We}=\frac{\rho u^{2}}{\frac{\sigma}{R_{\mathrm{m}}}},
$$

where $u$ is the relative approach velocity of the bubble walls, $\rho$ is the fluid density, $\sigma$ is the surface tension, and $R_{\mathrm{m}}$ is the mean bubble radius for which holds:

$$
\frac{2}{R_{\mathrm{m}}}=\frac{1}{R_{1}}+\frac{1}{R_{2}}
$$

We propose to extend the Weber number criterium to approaching walls of expanding bubbles. Then, for bubbles with a constant center-to-center distance, $u=\dot{R}_{1}+\dot{R}_{2}$. In our results, Weber numbers are found to be We $\gtrsim 1$ because of the rapid bubble expansions, with maximal radius increases of several $\mathrm{m} \mathrm{s}^{-1}$. If the Weber number is low, bubble coalescence will always occur, without flattening of the adjacent surfaces prior to contact (Chesters \& Hofman, 1982). In the 
high Weber number regime, coalescence is determined by a second step, after flattening: film drainage.

\section{Film drainage}

We investigate microbubble coalescence by computing the film drainage for no-slip (rigid) bubble surfaces resulting in a laminar flow, and for mobile (free) bubbles surfaces resulting in a plug flow.

Let us consider two bubbles with radii $R_{1}$ and $R_{2}$, and internal pressures $p_{1}$ and $p_{2}$, respectively, assumed spherical everywhere with the exception of a flattened interface that separates them through a liquid film of thickness $h$ ( $c f$. Fig. 3). The drainage rate of the liquid film depends on the difference $(p+\Pi)$ between the film pressure $p_{\mathrm{f}}$ and the liquid ambient pressure $p_{0}$. Here, $p$ is the difference in hydrodynamic pressure, while $\Pi$ is the disjoining pressure in the film. We estimate the pressure in the film by the mean of pressures $p_{1}$ and $p_{2}$, since the parallel film surfaces lead to equal pressure differences towards both bubbles:

$$
\begin{aligned}
p+\Pi=p_{\mathrm{f}}-p_{0}= & \frac{1}{2}\left(p_{1}+p_{2}\right)-p_{0}= \\
& =\sigma\left(\frac{1}{R_{1}}+\frac{1}{R_{2}}\right) \equiv p_{\mathrm{LY}}
\end{aligned},
$$

where $p_{\mathrm{LY}}$ is the Laplace-Young film pressure (Isenberg, 1992), and $\sigma$ is the surface tension. The disjoining pressure begins to slow down film thinning when $h$ drops below $0.1 \mu \mathrm{m}$, and becomes the dominant pressure term (usually owing to Van der Waals forces) when $h$ thins to about $10 \mathrm{~nm}$ (Marrucci, 1969; Chaudhari \& 
Hofmann, 1994; Bergeron, 1999). The eventual coalescence is very fast compared to the film drainage time scales considered later. Therefore, we neglect $\Pi$ and take $p$ equal to the Laplace-Young pressure for the films observed. As such, the pressure gradient determining the drainage velocity is independent of the ambient pressure.

We choose an $r-z$ coordinate system such that the film is symmetric around the plane $z=0$ and the line $r=0$, and that its boundaries are located at $z= \pm \frac{h}{2}$ and $r=R_{\mathrm{f}}$. The Laplace-Young pressure gradient drives liquid out of the film. The radial velocity of the liquid is described by a combination of a plug flow (present without any resistance to flow) and a laminar flow profile (in $z$ ) of Poiseuille-type induced by resistance at the film interfaces (Klaseboer et al., 2000; Young et al., 2000). The drainage of the liquid film can be parameterized by functions of these two contributions (Bazhlekov et al., 2000; Klaseboer et al., 2000; Hagesæther, 2002). We will study the two limiting cases of bubbles with no-slip interfaces and bubbles with free interfaces.

\section{No-slip interfaces}

In the presence of surfactant at sufficient surface concentration, the interfaces can be considered immobile (no-slip) (Lin \& Slattery, 1982b,a; Chen \& Slattery, 1982; van Wijngaarden, 1993). In the case of no-slip interfaces, the interfacial tangential velocity is zero, so the plug flow contribution is zero (Klaseboer et al., 
2000).

The film drainage velocity for rigid radial surfaces (disks) is given by the Reynolds equation (Reynolds, 1886; Sheludko, 1967; Kralchevsky et al., 1996):

$$
-\frac{\partial h}{\partial t}=\frac{2 p h^{3}}{3 \eta R_{\mathrm{f}}^{2}}
$$

where $\eta$ is the viscosity of the liquid, and $R_{\mathrm{f}}$ is the radius of the film surface.

The drainage time, $\tau_{\mathrm{d}}$, between the initial film thickness $h_{\mathrm{i}}$ and the critical film thickness $h_{\mathrm{c}}$ can be determined by integration of eq. (4):

$$
\int_{h_{\mathrm{i}}}^{h_{\mathrm{c}}}-\frac{\mathrm{d} h}{h^{3}}=\int_{0}^{\tau_{\mathrm{d}}} \frac{2 p}{3 \eta R_{\mathrm{f}}^{2}} \mathrm{~d} t .
$$

By taking $p$ and $R_{\mathrm{f}}$ constant over time, ${ }^{*}$ we obtain:

$$
\tau_{\mathrm{d}}=\frac{3 \eta R_{\mathrm{f}}^{2}}{4 p h_{\mathrm{c}}^{2}}\left(1-\frac{h_{\mathrm{c}}^{2}}{h_{\mathrm{i}}^{2}}\right)
$$

If $h_{\mathrm{c}}^{2} \ll h_{\mathrm{i}}^{2}$ the drainage time can be approximated by

$$
\tau_{\mathrm{d}} \approx \frac{3 \eta R_{\mathrm{f}}^{2}}{4 p h_{\mathrm{c}}^{2}}
$$

Figure 4 shows two no-slip drainage time curves as a function of equivalent

*Flattening takes place when:

$$
\dot{R}_{1}+\dot{R}_{2} \gg \frac{\partial h}{\partial t}
$$

whereas the flat film drainage happens in the next stage, when

$$
\dot{R}_{1} \approx \dot{R}_{2} \approx 0
$$

Thus, during drainage, we may take $p$ and $R_{\mathrm{f}}$ constant over time. 


$$
\begin{array}{rlc}
\eta & = & 0.001 \mathrm{Pas}^{2} \\
\rho & = & 998 \mathrm{~kg} \mathrm{~m}^{3} \\
\sigma & = & 0.072 \mathrm{~N} \mathrm{~m}^{-1} \\
\frac{2}{R_{\mathrm{e}}} & = & \frac{1}{R_{1}}+\frac{1}{R_{2}} \\
p & = & \frac{2 \sigma}{R_{\mathrm{e}}} \\
h_{\mathrm{c}} & = & 10 \mathrm{~nm} \text { or } 100 \mathrm{~nm} \\
\frac{h_{\mathrm{i}}}{h_{\mathrm{c}}} \approx & 100
\end{array}
$$

Table 1: Parameters used in the drainage equations.

bubble radius, using the parameters from Table 1 . Clearly, even for $h_{\mathrm{c}}=100 \mathrm{~nm}$, the drainage takes several microseconds for radii greater than $2 \mu \mathrm{m}$.

Even if the surfactant is very mobile, the interfaces have been observed to have a significant Poiseuille dissipation (Koehler et al., 2004). Drainage timescales for interfaces covered with mobile surfactant will therefore not be dramatically smaller than those indicated here for no-slip boundary conditions.

\section{Free interfaces}

In the case of free interfaces, the Poiseuille contribution to the drainage flow becomes negligible (van Wijngaarden, 1993; Klaseboer et al., 2000), and the drainage is inertial. The film drainage velocity for free radial surfaces is given 
by the equation (Kirkpatrick \& Lockett, 1974; Hagesæther, 2002):

$$
-\frac{\partial h}{\partial t}=\sqrt{\frac{8 p}{\rho}} \frac{h}{R_{\mathrm{f}}} .
$$

Note that the viscous term is absent. Similarly to the no-slip case, making the same quasi-static assumptions with regards to $p$ and $R_{\mathrm{f}}$, the drainage time can be approximated by

$$
\tau_{\mathrm{d}} \approx R_{\mathrm{f}} \sqrt{\frac{\rho}{8 p}} \log \left(\frac{h_{\mathrm{i}}}{h_{\mathrm{c}}}\right) .
$$

Figure 4 also shows two drainage time curves for free interfaces. These drainage times are much smaller than those for the no-slip situation, and depend only logarithmically on both the initial and the critical film thickness.

\section{Film rupture}

The disjoining pressure induces rupture by amplifying surface perturbations. These are initialized by either thermal fluctuations or by capillary waves Sharma \& Ruckenstein (1987). For thermal perturbations of a gas bubble in the micrometer range, the initial perturbation will be on the order of $\sqrt{\frac{k T}{\sigma}}$, where $k$ is Boltzmann's constant, and $T$ is the absolute temperature, in our situation approximately $300 \mathrm{~K}$. Hence, the initial thermal perturbation is lower than $1 \mathrm{~nm}$.

A film gradually thins to a critical thickness at which it either ruptures due to a local instability or at which it attains an equilibrium thickness. The mechanism of thin film rupture has been reviewed by Sheludko (1967). Angarska et al. (2004) 
measured these thicknesses in films, dependent of surfactant concentration and film radius. They found critical thicknesses in the range $20 \mathrm{~nm}<h_{\mathrm{c}}<40 \mathrm{~nm}$ for film radii $60 \mu \mathrm{m}<R_{\mathrm{f}}<160 \mu \mathrm{m}$.

For our film radii $\left(R_{\mathrm{f}}<10 \mu \mathrm{m}\right)$, we may assume critical thicknesses around $10 \mathrm{~nm}$, knowing that below $10 \mathrm{~nm}$ Van der Waals forces become very strong and rapid rupture of the film (and thus coalescence) ensues. Because of the weak dependence on film thickness, predictions from eq. (9) for coalescence time scales can be quite accurate even without precise knowledge of $h_{\mathrm{i}}$ and $h_{\mathrm{c}}$.

\section{Experimental setup}

Our experimental setup for imaging insonified contrast bubbles is as previously described by Postema et al. (2003). In short: a v389-su 500 kHz single-element transducer (Panametrics Inc., Waltham, MA) was mounted into a water-filled container, spherically focused at the focal plane of the optical system. The optical images were recorded through a microscope with an Imacon 468 fast framing camera (DRS Hadland, Ltd., Tring, UK), capable of taking 8 frames per experiment. It operated at a frame rate of 3 million frames per second (Mfps), corresponding to interframe times of $0.33 \mu \mathrm{s}$. Exposure times ranged from $10 \mathrm{~ns}$ to $70 \mathrm{~ns}$. The frames presented in this paper correspond to $30 \times 30 \mu \mathrm{m}^{2}$ areas, except for Figure 1(i). The first frame was typically captured prior to arrival of the 
ultrasound wave at the focal area. Seven frames were taken during ultrasound insonification, spanning a full ultrasound cycle of $2 \mu \mathrm{s}$. Contrast agent was insonified by 10 cycles of $0.5 \mathrm{MHz}$ ultrasound with high acoustic amplitudes, in the range $0.66-0.85 \mathrm{MPa}$.

We investigated coalescence events of an experimental UCA (Bracco Research SA, Geneva, Switzerland). According to manufacturer specifications, these bubbles are covered with a monolayer phospholipid shell and range in diameter from 1 to $6 \mu \mathrm{m}$ with a median of $2 \mu \mathrm{m}$. Undiluted UCA $(5 \mathrm{ml}$ of a $0.9 \% \mathrm{NaCl}$ dilution, added to a $25 \mathrm{mg}$ vial) was inserted through a cellulose capillary tube using either a syringe pressed by hand or a gravity fed or pumped infusion. This tube was positioned in the acoustic focus area. Since the capillary tube slightly moved within the acoustic focus area between experiments, the exact phase of the ultrasound wave in an image frame is not known. We performed 482 experiments at high acoustic amplitudes with the experimental UCA. We recorded 133 optical image sequences where microbubble coalescence was observed. Bubble sizes and distances were measured manually or by using a segmentation method described by Postema et al. (2003). 


\section{Results}

The observed phenomena are classified as follows: coalescence, bounce, multiple coalescence, and combined coalescence and fragmentation. In the following, we discuss representative image sequences typical for each process.

\section{Coalescence}

Figure 5(i) shows an example of coalescence. Figure 5(i)a shows three microbubbles with diameters (1) $2.5 \mu \mathrm{m}$, (2) $2.5 \mu \mathrm{m}$, and (3) $2.0 \mu \mathrm{m}$. After ultrasound arrival, microbubbles 2 and 3 have apparently coalesced (Fig. 5(i)b). The remaining bubble, with a diameter of $5.0 \mu \mathrm{m}$, is separated from microbubble 1 , which is seen to have expanded to $3.8 \mu \mathrm{m}$, with a center-to-center distance $d_{0}=4.8 \mu \mathrm{m}$. The center of bubble 1 has shifted slightly to the upper right. The thickness of the liquid film separating the bubble shells is approximately $h=1.1 \mu \mathrm{m}$, and the film radius is $1.7 \mu \mathrm{m}$. In Fig. 5(i)c the liquid film appears to have drained while the bubbles expanded, but, a separation is still visible. This boundary appears to have disappeared in Fig. $5(\mathrm{i}) \mathrm{d},{ }^{\dagger}$ leaving a pear-shaped bubble that turns ellipsoidal (Fig. 5(i)e) when contracting. Figure 5(i)f,g,h shows that the coalesced bubble expands uniformly. Because of the $3 \mathrm{Mfps}$ frame rate of the

\footnotetext{
${ }^{\dagger}$ If the line of sight is not perpendicular to the film, but at a tilt, the projection of the film boundaries might be obfuscated.
} 
image sequences, we conclude that the processes of drainage and rupture for the interposed film in Fig. 5(i)b take between $0.33 \mu$ s and $0.66 \mu \mathrm{s}$.

\section{Bounce}

Figure 5(ii) shows an example of bounce, i.e. bubbles approaching and flattening, but not coalescing. Figure 5(ii)a shows two microbubbles with diameters (1) $12.4 \mu \mathrm{m}$, and (2) $18.0 \mu \mathrm{m}$, much larger than those in Fig. 5(i)a. After ultrasound arrival, the bubble surfaces have flattened (Fig. 5(ii)b). The bubble centers have shifted towards each other. The film radius is $R_{\mathrm{f}}=4.8 \mu \mathrm{m}$. The film thickness is approximately $h=1.8 \mu \mathrm{m}$. In the remaining frames the bubbles expand and contract, but coalescence does not occur.

\section{Multiple coalescence}

Figure 5(iii) shows an example of multiple coalescence. In Fig. 5(iii)b an agglomerate of 8 touching microbubbles can be seen. Microbubbles 1 and 3 appear to be slightly above the focal plane (Postema et al., 2003). As the bubbles expand, they coalesce into one heart-shaped bubble and one ellipsoidal bubble (Fig. 5(iii)c,d). In the contraction phase, the heart-shaped bubble takes on an ellipsoidal shape (Fig. 5(iii)e). 


\section{Combined coalescence and fragmentation}

We observed repeated coalescence and fragmentation behavior in 12 events. Figure 5(iv) demonstrates the fragmentation, coalescence, and re-fragmentation of a microbubble. Figure 5(iv)a shows three microbubbles with diameters (1) $4.3 \mu \mathrm{m}$, (2) $2.6 \mu \mathrm{m}$, and (3) $2.8 \mu \mathrm{m}$. After ultrasound arrival, microbubbles 2 and 3 have translated towards microbubble 1 (Fig. 5(iv)b). From Fig. 5(iv)c, captured in contraction phase, it is appreciated that microbubble 1 has broken up into fragments. Three remaining fragments have started coalescing in Fig. 5(iv)d, and have obtained an irregular shape in Fig. 5(iv)e. The films separating the individual microbubbles have drained in Fig. 5(iv)f, while microbubble 2 appears to touch the coalescing structure. In Fig. 5(iv)g, the fragments of microbubble 1 have coalesced into one spherical bubble. Notice the translation of microbubble 2. Figure 5(iv)h shows that the coalesced bubble has re-fragmented during the compressive phase of the driving ultrasound.

\section{Discussion}

The variables obtained from Fig. 5(i)b are summarized in the first column of Table

2. For $h_{\mathrm{i}}$ we took the film thickness $h$ measured in this frame. If we substitute the parameters from Tables 1 and 2 into equation (9), we find a film drainage time $\tau_{\mathrm{d}}=0.35 \mu \mathrm{s}$ for free interfaces, consistent with the observed rapid coalescence 


\begin{tabular}{l|ll}
\hline & Fig. 5(i) & Fig. 5(ii) \\
\hline$R_{1}$ & $1.9 \mu \mathrm{m}$ & $6.2 \mu \mathrm{m}$ \\
$R_{2}$ & $2.5 \mu \mathrm{m}$ & $9.0 \mu \mathrm{m}$ \\
$R_{\mathrm{f}}$ & $1.7 \mu \mathrm{m}$ & $4.8 \mu \mathrm{m}$ \\
$h_{\mathrm{i}}$ & $1.1 \mu \mathrm{m}$ & $1.8 \mu \mathrm{m}$ \\
\hline
\end{tabular}

Table 2: Parameters measured in Fig. 5(i) and (ii).

in Fig. 5(i). For no-slip interfaces (7), even for a critical thickness as great as $h_{\mathrm{c}}=150 \mathrm{~nm}$, we find a film drainage time $\tau_{\mathrm{d}}=1.4 \mu \mathrm{s}$. Viscous drainage theory of thin films is thus insufficient to account for the observed coalescence.

The variables obtained from Fig. 5(ii)a,b are summarized in the second column of Table 2. For $h_{\mathrm{i}}$ we took the film thickness $h$ measured in Fig. 5(ii)b. If we use the parameters from the Table 1 and substitute the obtained parameters into equation (9) for free interfaces, $\tau_{\mathrm{d}}=2.0 \mu \mathrm{s}$. This is consistent with the absence of coalescence in Fig. 5(ii). Within $1 \mu$ s, the film can only drain to a minimum thickness $h_{\mathrm{m}}=0.13 \mu \mathrm{m}$. For no-slip interfaces (7), even for a critical thickness as great as $h_{\mathrm{c}}=150 \mathrm{~nm}$, we find a film drainage time $\tau_{\mathrm{d}}=39 \mu \mathrm{s}$, much longer than any timescale relevant to the observations.

The difference in drainage time scales between Fig. 5(i) and Fig. 5(ii) has a natural explanation in the different sizes of the bubbles involved in the process. As smaller films drain much faster, the relatively small bubbles of Fig. 5(i) coalesce 
over the experimental timescales, while the larger bubbles of Fig. 5(ii) are stable.

The results presented in Fig. 1 and Fig. 5 show that the bubbles have expanded to more than ten-fold their initial surface areas before coalescing. The UCA shell consists of a lipid monolayer that, under the conditions of our experiments, is in a solid state. It behaves like an elastic membrane that ruptures under relatively small strain (Zhou \& Joós, 1997). By the time of coalescence, therefore, the shell has ruptured, leaving newly formed clean free interfaces. Such interfaces will be on both sides of the film, and no surface dissipation can be expected here, so that the flow has to be (very near to) a plug flow, determined by a balance of inertia and driving pressure. This confirms that the interfaces may be assumed free and eq. (9) is applicable. We demonstrated previously with high-speed optical images that the UCA microbubbles used may expand to several times their original sizes, particularly at high acoustic pressures (Postema et al., 2003). The existence of stable holes of stretched lipid has been observed by Stine et al. (1990).

Our observations are incompatible with any drainage mechanism that involves resistance in either the bulk or the interfaces. The observed coalescence times agree with those computed for free interfaces, confirming that the expanded (snapped) lipid shells behave as if their surfaces are truly stress-free. The stretching and rupture of lipid membranes is currently under investigation at the Physics of Fluids group of the University of Twente.

Fragmentation may occur when the bubble interfaces are unstable, which is 
the case when the bubble is contracting and (inward) decelerating, i.e., when $\dot{R}<0 \wedge \ddot{R}>0$ (Plesset \& Mitchell, 1956; Brennen, 2002). Also, the formation of a re-entrant jet from a collapsing bubble has been related to the bubble shattering into fragments when the jet impacts the other side of the bubble surface. An optical image sequence of jet occurrence in a contrast microbubble was demonstrated by Postema et al. (2002b). Apparently water was projected through the freely flowing microbubble. Although we clearly observed jets in two events, we did not observe fragmentation in the same events (Postema et al., 2002a). We recently summarized the behavior of insonified UCA microbubbles (Postema et al., 2004b) including fragmentation. The number of fragments has been associated with the dominant spherical harmonic oscillation mode (Brennen, 2002).

Irregular shapes of oscillating bubbles, like those shown in Fig. 5(iii) and (iv), were interpreted as modes of shape instability of a single bubble before (Chomas et al., 1999a,b; May et al., 2001, 2002), but may also be accounted for by coalescence of bubbles or bubble fragments.

After coalescence, the resulting bubble will have acoustic properties different from those of the original bubbles, especially if its size is comparable to the size resonant with the ultrasonic driving. If small UCA microbubbles, having passed through the narrowest vessels, coalesce, they may be controlled to obtain resonant sizes. Especially for subharmonic imaging (Shankar et al., 1999), where twice 
the resonant bubble size is needed, and for tracking the diffusion of free gas bubbles with subharmonics, a promising technique in noninvasive blood pressure measurements (Postema et al., 2004a), controlled microbubble coalescence may be applicable. By the drainage timescales explained above, using the right ultrasound frequency could restrict coalescence to smaller bubbles, conveniently limiting the potentially dangerous coalescence of larger-radius contrast agents.

One of the effects of secondary radiation forces is that they lead to mutual attraction of similar-sized bubbles over multiple cycles. This may account for the translations observed in Fig. 5. Owing to secondary radiation forces, clusters of bubbles may be formed. By inducing coalescence of such groups of bubbles, and thus creating emboli, the perfusion of tumor vascularization may be reduced.

If coalescence of a lipid-shelled microbubble and a cell membrane can be induced, this will imply a promising technique in targeted drug delivery (van Wamel et al., 2002; Marmottant \& Hilgenfeldt, 2003).

\section{Conclusions}

Ultrasound-induced microbubble coalescence is the fusion of two or more microbubbles when subjected to an ultrasound field. Contrast agent microbubble coalescence has been observed frequently in an experimental setup. We showed that a coalescence mechanism for colliding bubbles also applies for expanding 
bubbles.

As adjacent bubbles expand, the following stages can be distinctly observed: flattening of the adjacent bubble surfaces prior to contact, drainage of the interposed liquid film toward a critical thickness, rupture of the liquid film, and formation of a single bubble. The time interval from flattening to coalescence was observed to take less than $1 \mu \mathrm{s}$ for ultrasound contrast agent microbubbles in the micron diameter range.

This fast coalescence cannot be explained by dissipation-limited film drainage rates. We conclude that the lipid shell ruptures upon expansion, exposing clean free bubble interfaces that support plug flow profiles in the film and inertia-limited drainage of which the timescales match those of the observed coalescence. At a given frequency, small bubbles are more prone to undergo coalescence than larger bubbles because of the smaller drainage timescales involved.

Ultrasound-induced coalescence has potential clinical applications in harmonic imaging, noninvasive blood pressure measurements, and targeted drug delivery.

\section{Acknowledgments}

This work has been supported by the Technology Foundation STW (RKG.5104). The authors are greatly indebted to Andrea Prosperetti for many fruitful 
discussions on coalescence theory.

\section{References}

Angarska JK, Dimitrova BS, Danov KD, Kralchevsky PA, Ananthapadmanabhan KP, Lips A. Detection of the hydrophobic surface force in foam films by measurements of the critical thickness of film rupture. Langmuir 2004; 20:1799-1806.

Bazhlekov IB, Chesters AK, van de Vosse FN. The effect of the dispersed to continuous-phase viscosity ratio on film drainage between interacting drops. Int J Multiphas Flow 2000;26:445-466.

Bergeron V. Forces and structure in thin liquid soap films. J Phys: Condens Matter 1999;11:R215-R238.

Brennen CE. Fission of collapsing cavitation bubbles. J Fluid Mech 2002; 472:153-166.

Burns PN, Hilpert P, Goldberg BB. Intravenous contrast agent for ultrasound Doppler: In vivo measurement of small tumor vessel dose-response. Proc Annu Int Conf IEEE Eng Med Biol Soc 1990;12:322-324.

Chaudhari RV, Hofmann H. Coalescence of gas bubbles in liquids. Rev Chem Eng 1994;10:131-190. 
Chen JD, Slattery JC. Effects of London-van der Waals forces on the thinning of a dimpled liquid film as a small drop or bubble approaches a horizontal solid plane. AIChE J 1982;28:955-963.

Chesters AK, Hofman G. Bubble coalescence in pure liquids. Appl Sci Res 1982; $38: 353-361$.

Chomas J, Dayton P, Allen J, Morgan K, Ferrara K. High speed optical experimental analysis of microbubble destruction, supported by theoretical development. Abstr 4th Eur Symp Ultrasound Contrast Imaging 1999a:52-55.

Chomas J, Dayton P, Morgan K, Allen J, Ferrara K. Optimization of microbubble destruction. Proc IEEE Ultrason Symp 1999b:1689-1692.

Chomas JE, Dayton P, May D, Ferrara K. Threshold of fragmentation for ultrasonic contrast. J Biomed Opt 2001;6:141-150.

de Jong N, Frinking PJA, Bouakaz A, Goorden M, Schuurmans T, Jingping X, Mastik F. Optical imaging of contrast agent microbubbles in an ultrasound field with a 100-MHz camera. Ultrasound Med Biol 2000;26:487-492.

Dhainaut M. Literature study on observations and experiments on coalescence and breakup of bubbles and drops. . Technical Report STF24 A02531, Trondheim: NTNU, Dept of Chemical Engineering 2002. 
Dimitrov DS, Ivanov IB. Hydrodynamics of thin liquid films. On the rate of thinning of microscopic films with deformable interfaces. J Colloid Interf Sci 1978;64:97-106.

Duineveld PC. Bouncing and coalescence of two bubbles in water. Enschede: PC Duineveld, 1994a.

Duineveld PC. Bouncing and coalescence phenomena of two bubbles in water. In: Blake JR, Boulton-Stone JM, Thomas NH, eds., Bubble Dynamics and Interface Phenomena, Dordrecht: Kluwer Academic Publishers, 1994b, volume 23 of Fluid mechanics and its applications. 447-456.

Goldberg BB, Raichlen JS, Forsberg F, eds. Ultrasound Contrast Agents. Basic principles and clinical applications. London: Martin Dunitz Ltd, 2001, 2nd edition.

Hagesæther L. Coalescence and break-up of drops and bubbles. Trondheim: Norwegian University of Science and Technology, 2002.

Isenberg C. The science of soap films and soap bubbles. Don Mills: General Publishing Company, Ltd, 1992, Dover edition.

Ivanov IB, Dimitrov DS, Radoyev BP. Generalized equations of thin films hydrodynamics and their application to the calculations of the thinning rate of films with non-deformed surfaces. Kolloid Zh 1979;41:36-42. 
Kirkpatrick RD, Lockett MJ. The influence of approach velocity on bubble coalescence. Chem Eng Sci 1974;29:2363-2373.

Klaseboer E, Chevaillier JP, Gourdon C, Masbernat O. Film drainage between colliding drops at constant approach velocity: experiments and modeling. J Colloid Interf Sci 2000;229:274-285.

Koehler SA, Hilgenfeldt S, Weeks ER, Stone HA. Foam drainage on the microscale. II. Imaging flow through single Plateau borders. J Colloid Interf Sci 2004:in press.

Kok JBW. Dynamics of a pair of gas bubbles moving through liquid. Part II. Experiment. Eur J Mech, B/Fluids 1993;12:541-560.

Kralchevsky PA, Danov KD, Ivanov IB. Thin liquid film physics. In: Prud'homme R, Khan S, eds., Foams, Theory, Measurements and Applications, New York: Marcel Dekker, 1996. 1-98.

Kudo N, Miyaoka T, Okada K, Yamamoto K, Niwa K. Study on mechanism of cell damage caused by microbubbles exposed to ultrasound. Proc IEEE Ultrason Symp 2002:1351-1354.

Kumaran V, Koch DL. The rate of coalescence in a suspension of high Reynolds number, low Weber number bubbles. Phys Fluids A 1993;5:1135-1140. 
Lin CY, Slattery JC. Thinning of a liquid film as a small drop or bubble approaches a fluid-fluid interface. AIChE J 1982a;28:786-792.

Lin CY, Slattery JC. Thinning of a liquid film as a small drop or bubble approaches a solid plane. AIChE J 1982b;28:147-156.

Marmottant P, Hilgenfeldt S. Controlled vesicle deformation and lysis by single oscillating bubbles. Nature 2003;423:153-156.

Marrucci G. A theory of coalescence. Chem Eng Sci 1969;24:975-985.

May D, Allen J, Ferrara K. Dynamics and fragmentation of thick-shelled microbubbles. IEEE Trans Ultrason, Ferroelect, Freq Contr 2002; 49:1400-1410.

May D, Allen J, Gut J, Ferrara K. Acoustic fragmentation of therapeutic contrast agents designed for localized drug delivery. Proc IEEE Ultrason Symp 2001:1385-1388.

Narsimhan G, Ruckenstein E. Structure, drainage, and coalescence of foams and concentrated emulsions. In: Prud'homme R, Khan S, eds., Foams, Theory, Measurements and Applications, New York: Marcel Dekker, 1996. 99-187.

Plesset MS, Mitchell TP. On the stability of the spherical shape of a vapor cavity in a liquid. Quart Appl Math 1956;13:419-430. 
Postema M, Bouakaz A, Chin CT, de Jong N. Optically observed microbubble coalescence and collapse. Proc IEEE Ultrason Symp 2002a:1900-1903.

Postema M, Bouakaz A, Chin CT, de Jong N. Simulations and measurements of optical images of insonified ultrasound contrast microbubbles. IEEE Trans Ultrason, Ferroelect, Freq Contr 2003;50:523-536.

Postema M, Bouakaz A, de Jong N. IEEE Trans Ultrason, Ferroelect, Freq Contr 2002b;49(3):cover.

Postema M, Bouakaz A, de Jong N. Noninvasive microbubble-based pressure measurements: a simulation study. Ultrasonics 2004a;42:759-762.

Postema M, van Wamel A, Lancée CT, de Jong N. Ultrasound-induced encapsulated microbubble phenomena. Ultrasound Med Biol 2004b; 30:827-840.

Reynolds O. On the theory of lubrication and its application to Mr. Beauchamp Tower's experiments, including an experimental determination of the viscosity of olive oil. Philos Trans Roy Soc A 1886;177:157-234.

Shankar PM, Krishna PD, Newhouse VL. Subharmonic backscattering from ultrasound contrast agents. J Acoust Soc Am 1999;106:2104-2110.

Sharma A, Ruckenstein E. Critical thickness and lifetimes of foams and 
emulsions: role of surface wave-induced thinning. J Colloid Interf Sci 1987; 119:14-29.

Sheludko A. Thin liquid films. Advan Colloid Interf Sci 1967;1:391-464.

Stine KJ, Rauseo SA, Moore BG, Wise JA, Knobler CM. Evolution of foam structures in Langmuir monolayers of pentadecanoid acid. Phys Rev A 1990; 41:6884-6893.

Takeuchi Y. IEEE Trans Ultrason, Ferroelect, Freq Contr 1999;46:cover.

van Wamel A, Bouakaz A, ten Cate F, de Jong N. Effects of diagnostic ultrasound parameters on molecular uptake and cell viability. Proc IEEE Ultrason Symp 2002:1387-1390.

van Wijngaarden L. The mean rise velocity of pairwise-interacting bubbles in liquid. J Fluid Mech 1993;251:55-78.

Young DF, Munson BR, Okiishi TH. A Brief Introduction to Fluid Mechanics. New York: John Wiley \& Sons, 2000, 2nd edition.

Zhou Z, Joós B. Mechanisms of membrane rupture: from cracks to pores. Phys Rev B 1997;56:29973009. 


\section{List of Tables}

1 Parameters used in the drainage equations. . . . . . . . . . . 9

2 Parameters measured in Fig. 5(i) and (ii). . . . . . . . . 16 


\section{List of Figures}

1 Optical images of stages of ultrasound-induced microbubble coalescence: (a) flattening of contact surfaces, (b) liquid film drainage, (c) forming of a merged bubble, (d) turning into an ellipsoidal bubble. Each frame in event (i) corresponds to a $21 \times 21 \mu \mathrm{m}^{2}$ area. Each frame in events (ii)-(iv) corresponds to a $30 \times 30 \mu \mathrm{m}^{2}$ area. Interframe times are $0.33 \mu \mathrm{s} . \ldots \ldots$

2 Schematic representation of stages of expanding bubble coalescence: (a) bubble collision, (b) flattening of contact surfaces, (c) liquid film drainage until a critical thickness (d), (e) film rupture, and (f) formation of an ellipsoidal bubble. . . . . . . 31

3 Schematic overview of variables used. . . . . . . . . . . 32

4 Drainage time as a function of equivalent bubble radius. . . . . . . 33

5 Optical images of (i) microbubble coalescence and spherical rebound, (ii) bounce, (iii) multiple coalescence, and (iv) repeated coalescence. Each frame corresponds to a $30 \times 30 \mu \mathrm{m}^{2}$ area. The frames in column a have been captured prior to ultrasound arrival. Interframe times are $0.33 \mu \mathrm{s} . \ldots \ldots \ldots . \ldots . \ldots 34$ 

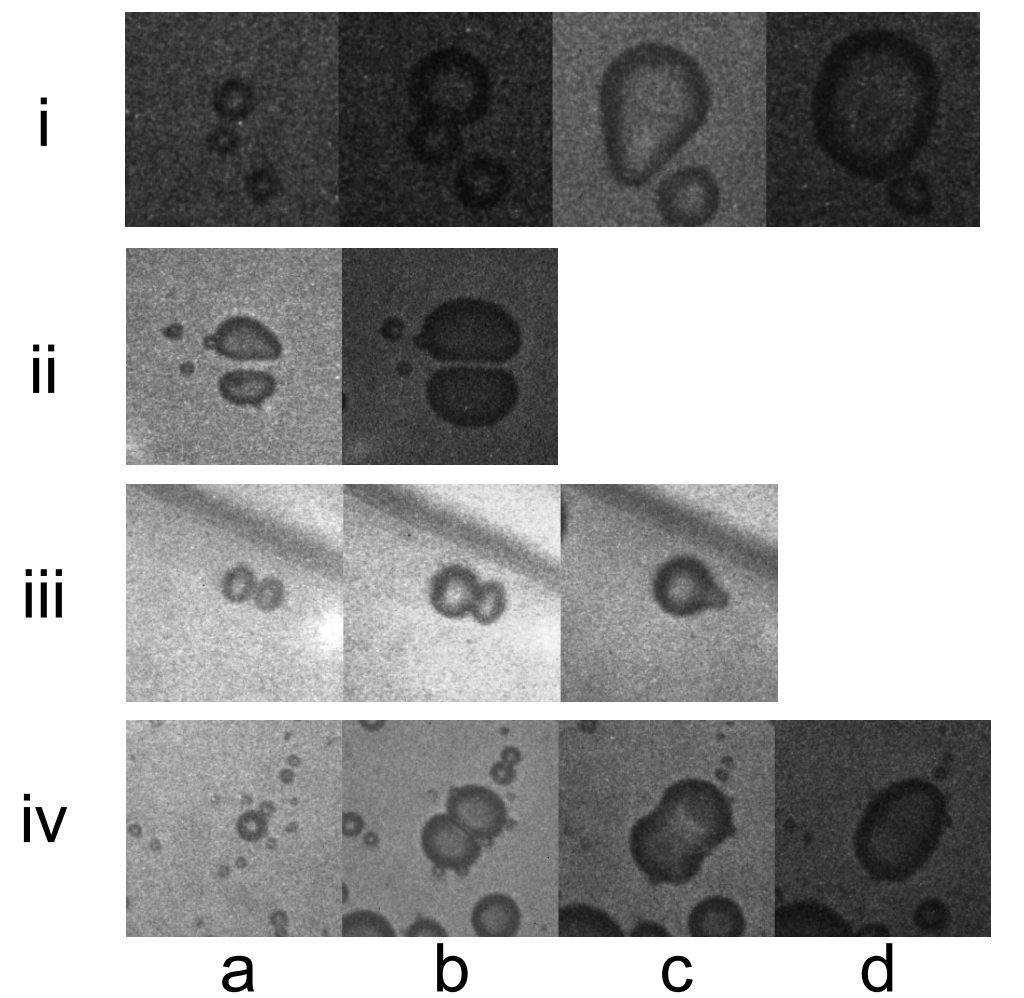

Figure 1: Optical images of stages of ultrasound-induced microbubble coalescence: (a) flattening of contact surfaces, (b) liquid film drainage, (c) forming of a merged bubble, (d) turning into an ellipsoidal bubble. Each frame in event (i) corresponds to a $21 \times 21 \mu \mathrm{m}^{2}$ area. Each frame in events (ii)-(iv) corresponds to a $30 \times 30 \mu \mathrm{m}^{2}$ area. Interframe times are $0.33 \mu \mathrm{s}$ 

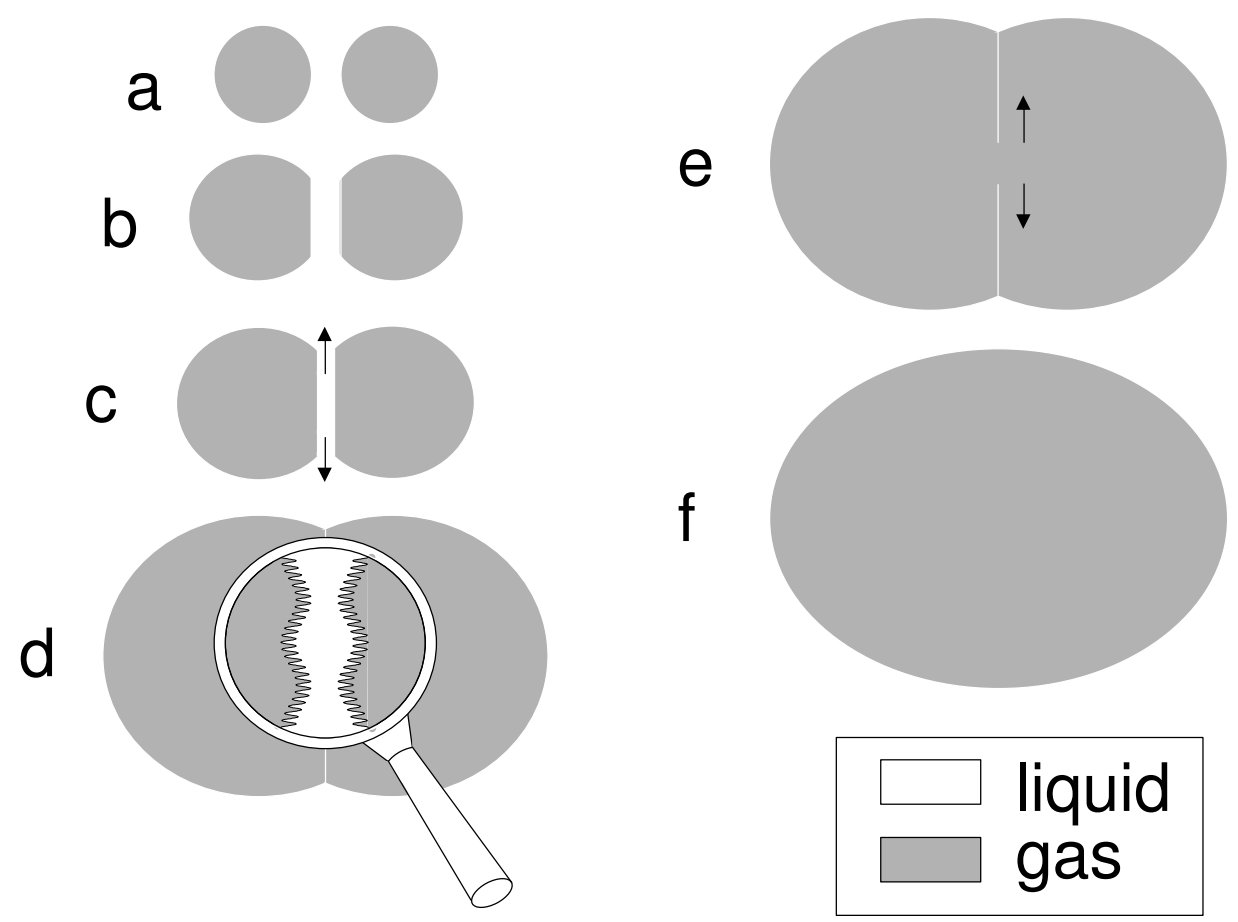

Figure 2: Schematic representation of stages of expanding bubble coalescence: (a) bubble collision, (b) flattening of contact surfaces, (c) liquid film drainage until a critical thickness (d), (e) film rupture, and (f) formation of an ellipsoidal bubble. 


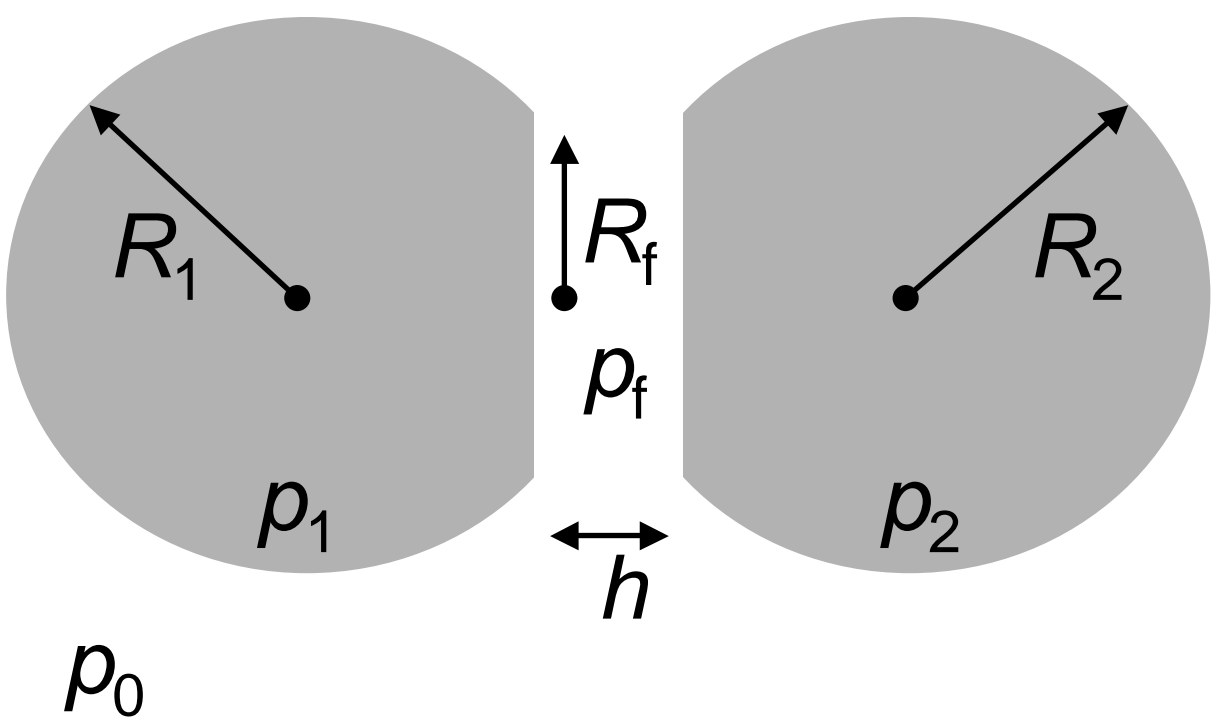

Figure 3: Schematic overview of variables used. 


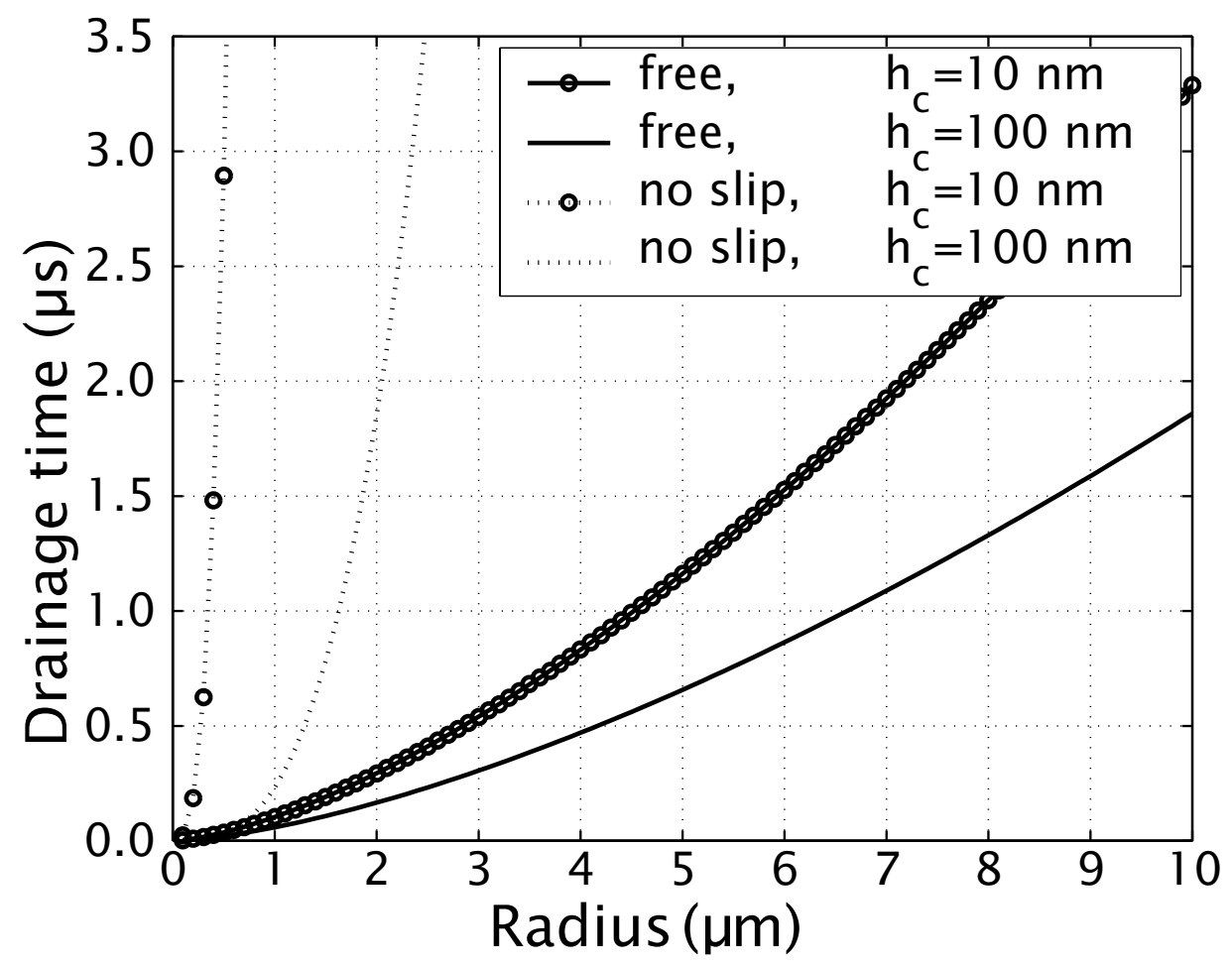

Figure 4: Drainage time as a function of equivalent bubble radius. 


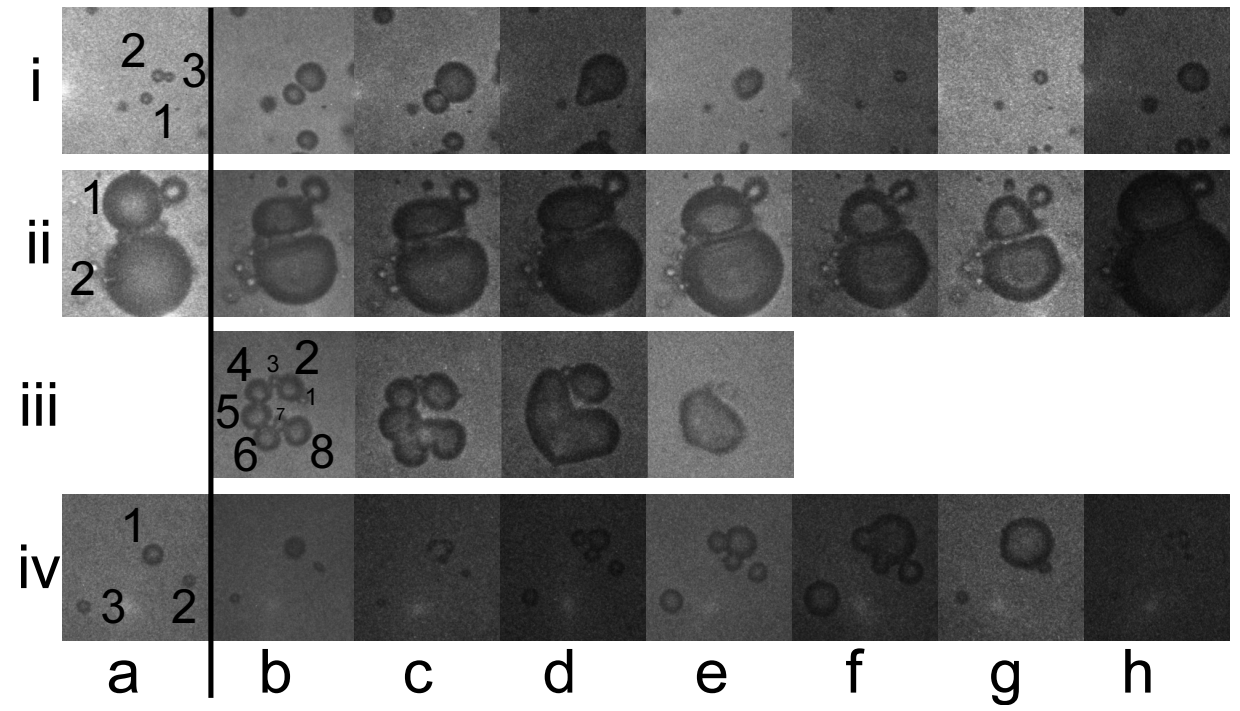

Figure 5: Optical images of (i) microbubble coalescence and spherical rebound, (ii) bounce, (iii) multiple coalescence, and (iv) repeated coalescence. Each frame corresponds to a $30 \times 30 \mu \mathrm{m}^{2}$ area. The frames in column a have been captured prior to ultrasound arrival. Interframe times are $0.33 \mu \mathrm{s}$. 